\title{
Fits to the spectra of broad-line region of active galactic nuclei and opacities for two-photon pair production
}

\author{
M. Ntshatsha* \\ Centre for Astro-Particle Physics (CAPP) and Department of Physics, University of \\ Johannesburg, Auckland Park 2006, South Africa \\ E-mail: mfuphin95@gmail.com

\section{S. Razzaque} \\ Centre for Astro-Particle Physics (CAPP) and Department of Physics, University of \\ Johannesburg, Auckland Park 2006, South Africa \\ E-mail: srazzque@uj.ac.za

\section{R.J. Britto} \\ University of the Free State, PO Box 339, Bloemfontein 9300, South Africa \\ E-mail: dr.richard.britto@gmail.com
}

\begin{abstract}
Based on the spectral analysis of a composite Hubble Space Telescope (HST) spectrum made of 184 quasar data, we model the emission lines of the broad-line region (BLR) using analytic functions. For simplicity of this paper we neglect effects of the continuum emission, which may be resulting from emission by the accretion disk, and assume the emission of the BLR to be composed of the most prominent emission lines that form as a result of ionization and recombination processes within regions of the BLR. We also calculate opacities for gamma-rays propagating in the BLR and interacting with the modeled emission to produce electron-positron $\left(e^{ \pm}\right)$pairs.
\end{abstract}

High Energy Astrophysics in Southern Africa - HEASA2018

1-3 August, 2018

Parys, Free State, South Africa

${ }^{*}$ Speaker. 


\section{Introduction}

Since their discovery in the 1960s [1] the superluminal properties of active galactic nuclei (AGN) have prompted astrophysicists to probe more into these objects. AGN are characterised into a variety of classes, however, the basic structure of all AGN is the same according to the unified model of AGN [2]. In the picture of the unified model is a supermassive black hole at the center of the AGN fueling the emission processes. Some radial distance away from the black hole is a torus of dusty/molecular clouds from which gas is being pulled by the gravitational force of the black hole. In the process of in-fall of the gas it accumulates as a thin disk of gas around the black hole, called the accretion disk or just simply disk. Across a large radial range of the disk, wind outflow processes from the disk contribute to the formation of the BLR close to the disk [3], (a distance in the order of light years from the black hole [4]) . Further away from the outer edge of the BLR, where there exists an apparent gap, is the narrow line region (NLR) [5]. There are clear distinctions between the BLR and the NLR, observable mainly by considering the full width at half maximum (FWHM) of their spectral lines ( $\sim 4000 \mathrm{~km} / \mathrm{s}$ and $\sim 500 \mathrm{~km} / \mathrm{s}$, respectively [5]). Both the BLR and NLR radii, $\left(R_{B L R}, R_{N L R}\right)$, scale as $\sim L^{1 / 2}$, where $L$ is the luminosity of the central source. However, they do differ in radius and covering factor where $R_{N L R} \sim(10-100)$ pc with covering factor of about $2 \%$ [5], in comparison to $R_{B L R} \sim(0.1-1)$ pc [4] with a covering factor of (5-30)\% [5].

The $\gamma$-ray spectra of quasars can be typically described by a simple power-law, however, in some blazar spectra they break away from the trend of the power-law at certain $\gamma$-ray energies. With these breaks at specific energies, models such as the broken power-law or log-parabola then become better functions that physically describe both ends of the spectrum at enegies lower and higher than this break energy [4]. It was shown by [4,6] that the break energies in some blazar spectra are consistent with the absorption of $\gamma$-ray photons by some emission lines in the BLR through $e^{ \pm}$pair production. To calculate this absorption detailed information about the distribution of the target photon gas is required [7].

In this proceedings we investigate the absorption of $\gamma$-rays propagating through the BLR in bright AGN. We model the BLR by fitting the most visible emission lines obtained from the composite spectrum of HST [8]. By modeling the BLR in this way we aim for a more realistic description of its photon distribution across its entire emission range. Finally we calculate the opacity of the BLR for $\gamma$-rays in the energy range $90 \mathrm{MeV}-400 \mathrm{GeV}$.

\section{Data Analysis}

We use the HST data of a composite spectrum of 332 spectra of 184 quasars composed of both radio loud and radio quiet AGN. We plotted data ${ }^{1}$ from Figure 1 of [8] by converting the relative flux $F_{\lambda}$ in the $(303-4989) \AA$ wavelength range into a $F_{\varepsilon}$ relative flux in the $(2.48-41.0) \mathrm{eV}$ energy range. In the composite HST quasar data composed and studied by [1] they showed that in the wavelength range $(350-3000) \AA$ there is a distinct change in the shape of the continuum. They reported power law indices of $\sim-2$ in the extreme ultraviolet $(E U V)$ region $\sim(300-1052) \AA$ and $\sim-1$ in the near ultraviolet $(\mathrm{NUV})$ region $\sim(1052-3000) \AA$. With this change in the power law index they concluded that the quasar UV spectral continuum can be approximated with a broken

\footnotetext{
${ }^{1}$ ASCII file provided by R. C. Telfer
} 
power law (BPL). In the work of [8] they show that the same pattern is observed for subset samples of radio loud and radio quiet quasars. In fitting the continuum of the composite spectrum we then followed $[1,8]$ by fitting it with a broken power law $\propto\left(\varepsilon / \varepsilon_{B}\right)^{-\beta_{j}}$ with $j=1,2$ below and above the break energy $\varepsilon_{B}$ respectively. However, instead of fitting it using the IRAF task specfit in the wavelength windows chosen in [8], we rather "hand"-fitted the continuum over almost the entire UV range of the HST data file , $(303$ - 4989) $\AA$, where we have excluded 12 data points (291 302) $\AA$ which had large errors associated with them. In so doing we obtained power-law indices $\beta_{1}=-1.35$ and $\beta_{2}=-0.18$ below and above the break energy $\varepsilon_{B}=9.8 \mathrm{eV}$ respectively. We note here that at the low wavelengths $(<560 \AA)$ the composite spectrum is composed of less than 10 spectra contributing to the composite at a given wavelength, as it is mentioned in [8]. From this point $(<560 \AA)$ there is a slight hardening of the continuum, deviating from the power law [8]. It is noteworthy to mention that the small number of contributing spectra in this wavelength range may be the cause of this deviation and for this reason the hardening here may not necessarily be characteristic of all quasars [8]. The emission lines in this region, however, are real and reflect high signal to noise ratio data obtained to study the He II Gunn-Peterson effect [8]. Above $900 \AA$, any deviation from the power law is caused by weak emission features, particularly Fe II [1]. At wavelengths above $2200 \AA$ these features are most prominent that they cause the continuum shape to deviate quite significantly from the power law. However, in this proceedings we attempt to aim for the full representation of the BLR across all its emission. To achieve this we performed a fit over the wavelength range (303 - 4989) $\AA$, effectively representative of the whole UV range of the BLR spectrum of quasars.

To fit the BLR emission lines it was convenient to remove the continuum by subtracting the fitted BPL from the data. Each emission line from a given frequency range was isolated and independently fit with either the Breit-Wigner (BW) for lines with a narrow profile, the Gaussian (Ga) for lines with a broad profile or a combination of the two if the profile of a line had prominent broad and narrow components. The emission lines are each fitted individually, however, due to the high density of some emission features in the range below the peak labeled $\lambda 2085$, the so-called $3000 \AA$ bump $(\approx 2200-4000) \AA$ [9], we use the Smoothly-joint Broken Power Law (SBPL) as an average estimate of the combined emission of the lines appearing in the energy range $(3.35-4.09) \mathrm{eV}$, labeled as $\lambda_{B} 3205$ and $(4.60-5.50) \mathrm{eV}$ labeled $\lambda_{B} 2459$. Other ranges averaged over with the SBPL are $(18.5-20.6) \mathrm{eV}$ labeled $\lambda_{B} 640,(22.1-26.8) \mathrm{eV} \lambda_{B} 474,(28.1-34.6) \mathrm{eV} \lambda_{B} 404$ and (34.639.4) $\mathrm{eV} \lambda_{B} 328$, where the subscript $B$ signifies the position of the break chosen in the SBPL. It is not the position of the specific line. Many of the lines fitted in this proceedings are not identified, especially below the Lyman limit (912 $⿱$ ) ) and although the composite spectrum considered here is representative of the BLR behaviour of a typical quasar, it may not necessarily be the case that all the lines considered in this proceedings will appear in all quasars. The composite simply suggests hints of the existence of these lines, which we consider for completeness and without loss of generality. The functions used for fitting are expressed as 


$$
\begin{aligned}
B W(\varepsilon) & =\frac{n_{i}}{2 \pi} \frac{w_{i}}{\left(\varepsilon-\varepsilon_{i}\right)^{2}+\left(w_{i} / 2\right)^{2}} \\
S B P L(\varepsilon) & =\frac{n_{i}}{\varepsilon_{i}}\left[\left(\frac{\varepsilon}{\varepsilon_{i}}\right)^{\alpha_{1}}+\left(\frac{\varepsilon}{\varepsilon_{i}}\right)^{\alpha_{2}}\right]^{-1} \\
G a(\varepsilon) & =\frac{n_{i}}{\varepsilon_{i}} \exp \left[-\left(\frac{\varepsilon-\varepsilon_{i}}{\sqrt{2} w_{i}}\right)^{2}\right]
\end{aligned}
$$

where

$$
n_{i}\left[\mathrm{~cm}^{-3}\right]=1.66 \times 10^{11}\left(\frac{R_{B L R}}{10^{17} \mathrm{~cm}}\right)^{-2}\left(\frac{\varepsilon_{i}}{\mathrm{eV}}\right)^{-1}\left(\frac{k_{i} L_{\mathrm{Ly}_{\alpha}}}{10^{45} \mathrm{erg} / \mathrm{s}}\right)
$$

$k_{i}$ is the fraction of the luminosity of the $i^{\text {th }}$ line relative to the luminosity of the $\mathrm{Ly}_{\alpha}$ line. $L_{\mathrm{Ly}_{\alpha}}$ in the above equations is the luminosity of $\mathrm{Ly}_{\alpha}$. The $\mathrm{Ly}_{\alpha}$ line was fitted as a linear combination of the $\mathrm{BW}$ and $\mathrm{Ga}$, the $k_{i}$ for the $\mathrm{Ga}$ component was taken to be $33 \%$ that of the $\mathrm{BW}$ component. All the lines in the fits are then normalized by the $k_{i}$ of the BW component of $\mathrm{Ly}_{\alpha} . \varepsilon_{i}$ is the peak energy of the $i^{t h}$ line and $w_{i}$ is the energy width of the line. $n_{i}$ is the density of photons emitted by the $i^{\text {th }}$ line, where we make the crude assumption that the emission lines appear at the same radius within the BLR, so that there is no variation in photon density. In reality, different emission lines originate from different regions of the BLR and the photon density varies with radius. A detailed calculation in future will need to include this effect while calculating the $\gamma$-ray opacity. The indices $\alpha_{1}$ and $\alpha_{2}$ describe the steepness of the rise and fall of a line before and after the peak energy $\varepsilon_{i}$ respectively. To estimate the size of the BLR we use its scaling $R_{B L R} \approx 10^{18} L_{d i s k, 47}^{1 / 2} \mathrm{~cm} \mathrm{[6]}{ }^{2}$. Since the BLR reprocesses a fraction of the disk emission in both its lines and continuum [10] we take the luminosity of the BLR, $L_{B L R}=0.1 L_{\text {disk }}$ to be a fraction of the disk luminosity [11] as it is treated in one of the cases in the works of [10]. From Table 1 of [8] we get the average disk luminosity of $10^{46.16} \mathrm{erg} / \mathrm{s}$ corresponding to a BLR size of $R_{B L R} \approx 12.02 \times 10^{17} \mathrm{~cm}$ for the composite spectrum. The peak energies $\varepsilon_{i}$ of most of the lines were obtained from Table 1 in [8] and kept fixed, but in some cases we needed to shift them to match the data. The equivalent widths of the lines were also held fixed and all the values (with the exception of the Ga component in the $\mathrm{Ly}_{\alpha}$ fit) of the parameter $k_{i}$ used in the fits were kept free and obtained using the scipy.optimize.curve_fit optimization package in python. The indices $\left(\alpha_{1}, \alpha_{2}\right)$ were held fixed for lines and regions where curve fit gave either infinities for the errors in the parameters, or error values larger than the parameters. Instances where the indices were free are indicated with an asterisk in Table 1. The scipy.optimize.curve_fit function optimizes and determines fit parameters of a given model by using least squares minimization. To get a quantitative measure of the goodness of the fit we calculated a reduced $\chi_{\text {red }}^{2}$ on each trial. The lowest $\chi_{\text {red }}^{2}$ obtained was 4.2 with 4639 degrees of freedom for the fit presented here. The fit parameters together with the model used are tabulated in Table 1. Figure 1 a) shows the plot (in red) of the fit imposed on the BLR composite data, where Figure $1 \mathrm{~b}$ ) in the bottom panel is a plot of the residual calculated as shown in the figure.

Next we calculate the opacities of $e^{ \pm}$pair creation by $\gamma$-rays interacting with the BLR photons. We used the standard opacity formula from [12] and adapted it for the fitting functions as:

\footnotetext{
${ }^{2}$ In this notation $L_{d i s k}=10^{47} L_{d i s k, 47} \mathrm{erg} / \mathrm{s}$ is the accretion disk luminosity.
} 
Table 1: Parameter list and model used in fitting the spectral lines of the quasar spectrum.

\begin{tabular}{|c|c|c|c|c|c|c|}
\hline $\begin{array}{c}\text { Line / } \\
\text { energy range }\end{array}$ & Function & $k_{i}$ & $\begin{array}{c}\text { Line peak } \\
\text { energy } \varepsilon_{i}[\mathrm{eV}] \\
\text { (Fixed) }\end{array}$ & $\begin{array}{c}\text { Line width } \\
w_{i}[\mathrm{eV}] \\
\text { (Fixed) }\end{array}$ & $\begin{array}{c}\alpha_{1} \\
\text { (Fixed) }\end{array}$ & $\begin{array}{c}\alpha_{2} \\
\text { (Fixed) }\end{array}$ \\
\hline 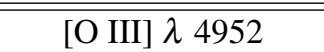 & $\overline{\mathrm{BW}}$ & $1.41 \times 10^{-4}$ & 2.50 & 0.008 & & \\
\hline $\mathrm{H} \beta \lambda 4857$ & BW & $4.18 \times 10^{-4}$ & 2.55 & 0.02 & & \\
\hline $\mathrm{H} \gamma \lambda 4330$ & BW & $2.90 \times 10^{-5}$ & 2.86 & 0.003 & & \\
\hline$[\mathrm{Ne}$ III $] \lambda 3862$ & BW & $1.70 \times 10^{-4}$ & 3.21 & 0.009 & & \\
\hline$[\mathrm{O}$ II] $\lambda 3723$ & BW & $7.71 \times 10^{-4}$ & 3.33 & 0.03 & & \\
\hline$\lambda_{B} 3205$ & SBPL & $3.15 \times 10^{-1}$ & 3.87 & & 13.2 & -12.8 \\
\hline Fe II $\lambda 2959$ & BW & $2.53 \times 10^{-2}$ & 4.19 & 0.36 & & \\
\hline $\operatorname{Mg}$ II $\lambda 2799$ & $\begin{array}{l}\mathrm{BW} \\
\mathrm{Ga}\end{array}$ & $\begin{array}{l}8.29 \times 10^{-3} \\
3.03 \times 10^{-1}\end{array}$ & $\begin{array}{l}4.43 \\
4.43\end{array}$ & $\begin{array}{c}0.056 \\
0.14\end{array}$ & & \\
\hline$\lambda_{B} 2459$ & SBPL & $5.14 \times 10^{-1}$ & 5.04 & & 15.2 & -8.8 \\
\hline Fe II $\lambda 2225$ & $\mathrm{Ga}$ & $6.47 \times 10^{-2}$ & 5.57 & 0.25 & & \\
\hline Fe III $\lambda 2085$ & BW & $8.79 \times 10^{-3}$ & 5.95 & 0.25 & & \\
\hline C III + Si III] & BW & $3.04 \times 10^{-2}$ & 6.50 & 0.09 & & \\
\hline$\lambda 1907$ & $\mathrm{Ga}$ & $2.66 \times 10^{-1}$ & 6.50 & 0.2 & & \\
\hline $\mathrm{N}$ III] $\lambda 1749$ & BW & $1.40 \times 10^{-2}$ & 7.09 & 0.16 & & \\
\hline $\begin{array}{c}\lambda 1638 \\
\text { possibly He II }\end{array}$ & BW & $9.07 \times 10^{-2}$ & 7.57 & 0.32 & & \\
\hline C IV $\lambda 1549$ & $\begin{array}{l}\mathrm{BW} \\
\mathrm{Ga}\end{array}$ & $\begin{array}{c}1.87 \times 10^{-1} \\
2.25\end{array}$ & $\begin{array}{l}8.00 \\
8.00\end{array}$ & $\begin{array}{c}0.0995 \\
0.19\end{array}$ & & \\
\hline Si IV + O IV] $\lambda 1399$ & BW & $7.02 \times 10^{-2}$ & 8.86 & 0.173 & & \\
\hline C II $\lambda 1335$ & BW & $3.64 \times 10^{-3}$ & 9.29 & 0.041 & & \\
\hline O I + Si II $\lambda 1304$ & BW & $2.43 \times 10^{-2}$ & 9.51 & 0.12 & & \\
\hline N V $\lambda 1240$ & BW & $1.50 \times 10^{-1}$ & 10.0 & 0.129 & & \\
\hline Ly $\alpha \lambda 1215$ & $\begin{array}{l}\mathrm{BW} \\
\mathrm{Ga}\end{array}$ & $\begin{array}{c}1 \\
0.33\end{array}$ & $\begin{array}{l}10.20 \\
10.20\end{array}$ & $\begin{array}{l}0.14 \\
0.82\end{array}$ & & \\
\hline Fe III $\lambda 1123$ & BW & $2.73 \times 10^{-2}$ & 11.04 & 0.18 & & \\
\hline $\operatorname{Ar} I \lambda 1064$ & BW & $1.71 \times 10^{-1}$ & 11.65 & 0.42 & & \\
\hline $\mathrm{O}$ VI + Ly $\beta \lambda 1032$ & BW & $4.15 \times 10^{-1}$ & 12.01 & 0.27 & & \\
\hline C III + N III $\lambda 983$ & BW & $2.77 \times 10^{-1}$ & 12.61 & 0.48 & & \\
\hline $\begin{array}{c}\mathrm{H} \text { I + Ly series +S VI } \\
\lambda_{B} 940\end{array}$ & SBPL & 5.05 & 13.19 & & 42.77 & -49.84 \\
\hline O III $\lambda 837$ & $\mathrm{BW}$ & $4.69 \times 10^{-2}$ & 14.81 & 0.2 & & \\
\hline $\mathrm{Ne}$ VIII + O IV $\lambda 783$ & SBPL & 8.19 & 15.83 & & 59.99 & -59.97 \\
\hline$\lambda 702$ & BW & $2.11 \times 10^{-1}$ & 17.66 & 0.594 & & \\
\hline$\lambda 685$ & BW & $2.85 \times 10^{-1}$ & 18.1 & 0.7 & & \\
\hline$\lambda_{B} 640$ & SBPL & 12.03 & 19.4 & & 37.12 & -37.10 \\
\hline$\lambda 599$ & BW & $1.40 \times^{-1}$ & 20.7 & 0.3 & & \\
\hline$\lambda 576$ & BW & $3.74 \times 10^{-1}$ & 21.53 & 0.594 & & \\
\hline$\lambda_{B} 474$ & SBPL & 32.81 & 26.16 & & $64.83^{*}$ & $-26.40 *$ \\
\hline$\lambda 457$ & BW & 1.03 & 27.1 & 0.91 & & \\
\hline$\lambda_{B} 404$ & SBPL & 91.84 & 30.69 & & $11.28 *$ & $-12.03 *$ \\
\hline$\lambda_{B} 328$ & SBPL & 144.37 & 37.80 & & $30.31^{*}$ & $-17.53 *$ \\
\hline$\lambda 313$ & BW & $6.80 \times 10^{-1}$ & 39.61 & 0.093 & & \\
\hline$\lambda 309$ & BW & 2.65 & 40.12 & 0.594 & & \\
\hline $\mathrm{He}_{\mathrm{II}} \mathrm{Ly}_{\alpha} \lambda 305$ & BW & 3.33 & 40.65 & 0.356 & & \\
\hline
\end{tabular}



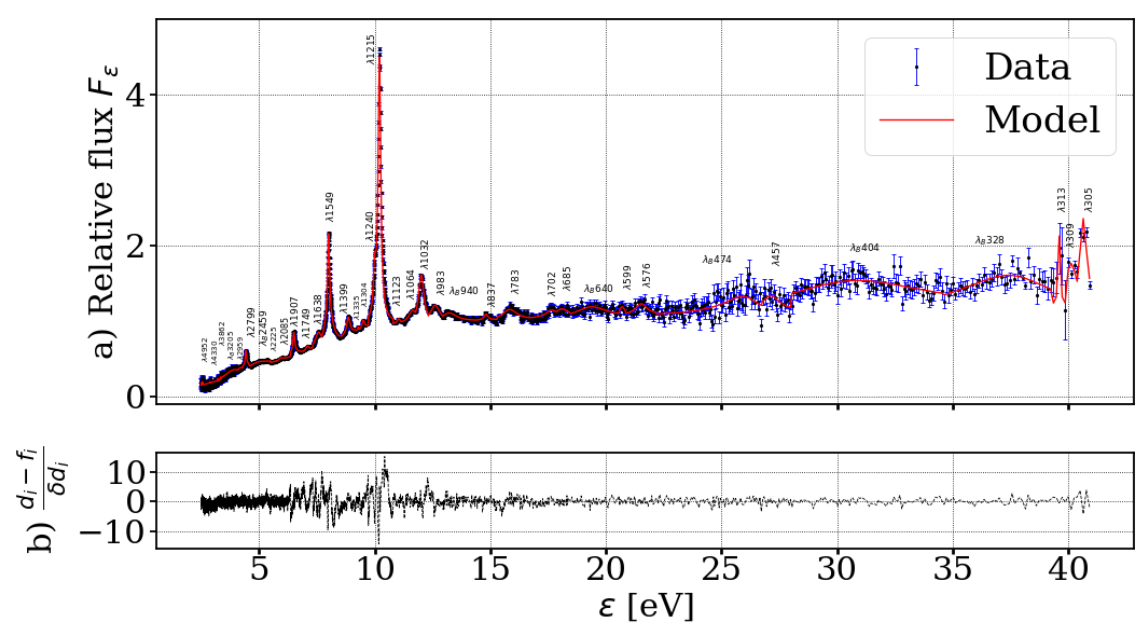

Figure 1: a) Fit of the emission lines extending from $(2.48-41.0) \mathrm{eV}$ of the BLR spectrum (solid red line). b) Plot of the residual at each $\varepsilon$. On the y-axis of the bottom panel the symbols $d_{i}, f_{i}, \delta d_{i}$ are the $i^{\text {th }}$ data point, model evaluated at the $i^{\text {th }}$ point and the error on the $i^{t h}$ data point, respectively.

$$
\begin{aligned}
\frac{d \tau_{B W}}{d x} & =\frac{r_{0}^{2}}{2}\left(\frac{m_{e}^{2} c^{4}}{E(1+z)}\right)^{2} \sum_{i} n_{i} w_{i} \int_{\frac{m_{e}^{2} c^{4}}{E(1+z)}}^{\infty} \frac{\bar{\phi}\left[s_{0}(\varepsilon)\right]}{\varepsilon^{2}\left[\left(\varepsilon-\varepsilon_{i}\right)^{2}+\left(w_{i} / 2\right)^{2}\right]} d \varepsilon \\
\frac{d \tau_{S B P L}}{d x} & =1.3 \pi r_{0}^{2}\left(\frac{m_{e}^{2} c^{4}}{E(1+z)}\right)^{2} \sum_{i} \frac{n_{i}}{\varepsilon_{i}} \int_{\frac{m_{e}^{2} c^{4}}{E(1+z)}}^{\infty} \frac{\bar{\phi}\left[s_{0}(\varepsilon)\right]}{\varepsilon^{2}\left[\left(\varepsilon / \varepsilon_{i}\right)^{\alpha_{1}}+\left(\varepsilon / \varepsilon_{i}\right)^{\alpha_{2}}\right]} d \varepsilon \\
\frac{d \tau_{G a}}{d x} & =1.5 \pi r_{0}^{2}\left(\frac{m_{e}^{2} c^{4}}{E(1+z)}\right)^{2} \sum_{i} \frac{n_{i}}{\varepsilon_{i}} \int_{\frac{m_{(1}^{2} c^{4}}{E(1+z)}}^{\infty} \frac{\bar{\phi}\left[s_{0}(\varepsilon)\right]}{\varepsilon^{2} \exp \left[\left(\frac{\varepsilon-\varepsilon_{i}}{\sqrt{2} w_{i}}\right)^{2}\right]} d \varepsilon
\end{aligned}
$$

where equation (2.5) is taken from [11], the factors in front of equations (2.6), (2.7) are the ratios of the area under the curves BW/SBPL and BW/Ga, and $n_{i}$ is the quantity referred to by equation (2.4), $\bar{\phi}[v]=\int_{1}^{v} s \bar{\sigma}_{\gamma \gamma}(s) d s, s_{0}(\varepsilon)=\varepsilon E / m_{e}^{2} c^{4}, \bar{\sigma}_{\gamma \gamma}=1 / s\left[\left(2+2 / s-1 / s^{2}\right) \ln \frac{1+\beta}{1-\beta}-2 \beta(1+1 / s)\right]$ is the dimensionless photon cross-section where $\beta^{2}=(1-1 / s), m_{e}$ is the elemental electron mass, $r_{0}$ is the classical electron radius, $z$ is the redshift and $\varepsilon, E$ are the target photon, gamma photon energies respectively. We calculate the opacities by taking the radius of the BLR as $R_{B L R}=12.02 \times 10^{17} \mathrm{~cm}$ as inferred by [6]. The opacities of the fitted emission lines are shown in Figure 2, with the blue dot-dash line being the sum of the line opacities.

In the delta function approximation for $\gamma \gamma$ absorption the opacity is given by:

$$
\tau_{\gamma \gamma}\left(L_{i}, \varepsilon_{i}\right)=\frac{L_{i}}{4 \pi c R_{B L R} \varepsilon_{i}} \frac{\sigma_{T}}{5} \approx 16.5\left(\frac{L_{i}}{9.2 \times 10^{44} \mathrm{erg} / \mathrm{s}}\right)\left(\frac{R_{B L R}}{1.2 \times 10^{18} \mathrm{~cm}}\right)^{-1}\left(\frac{\varepsilon_{i}}{10.2 \mathrm{eV}}\right)^{-1}
$$

which is the opacity for the $\operatorname{Ly}_{\alpha}$ line, $L_{i}$ and $\sigma_{T}$ are the line luminosity and the Thomson crosssection, respectively. 


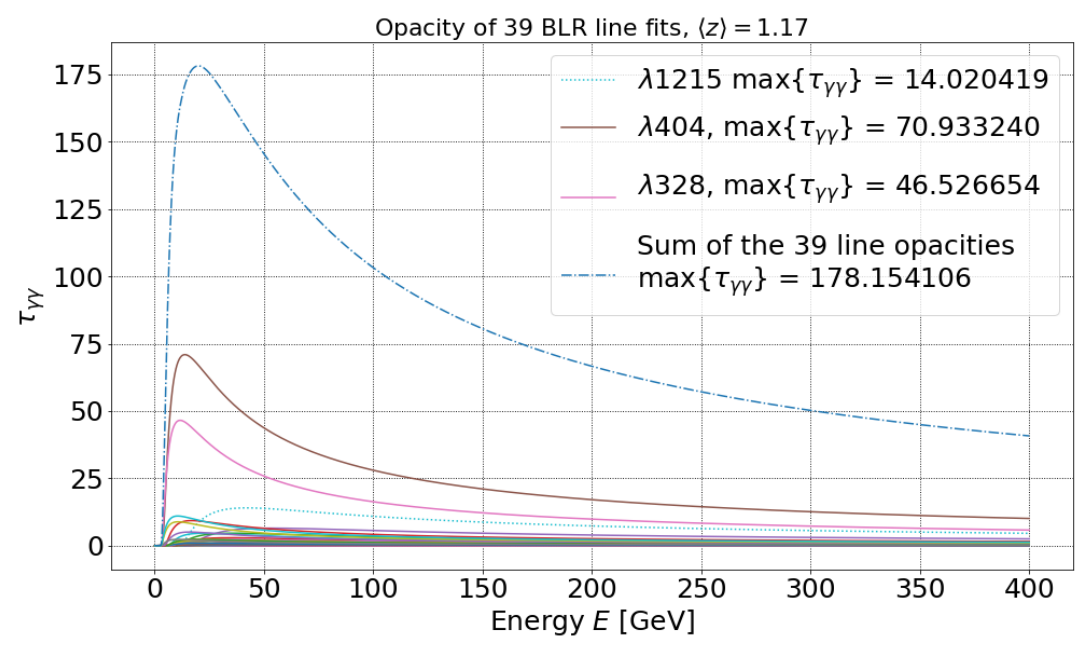

Figure 2: Opacity of BLR lines identified in the composite spectrum of 184 quasars. The blue dot-dash line is the sum of the individual line opacities.

\section{Summary and Discussion}

The careful analysis of a large sample of quasar spectra spanning a large range in redshift $0.33<$ $z<3.7$ done by [8] has made it possible to model the emission of the BLR lines. The BW, SBPL and $\mathrm{Ga}$ functions were used to individually fit the peaks of the quasar data and the data was well reproduced by the fits with $\chi_{\text {red }}^{2}=4.2$. The modeling of these lines gives a reasonable description of the BLR and a good opacity estimate of $\gamma$-ray photons in the BLR. The maximum opacity summing of all the emission lines came to the value of $\tau \sim 178$ at $\sim 18.9 \mathrm{GeV}$. This threshold energy is nearly consitent with that of the $20 \mathrm{GeV}$ breaks due to $\mathrm{H} \mathrm{LyC}$ in many blazars reported by [6]. At $\tau \sim 178$, the opacity sum of the whole composite is quite high and this is due to the high density of the emission lines in the EUV which are averaged over with the SBPL, in particular the $\lambda_{B} 404$ and $\lambda_{B} 328$ regions. And as it has already been hinted in section 2 by a slight hardening of the continuum at low wavelengths, this behaviour may not necessarily be representative of all quasars since only few quasar spectra contributed to the composite spectrum at these wavelengths. However, if this slight hardening of the spectrum at these wavelengths happens to be real in a typical quasar, then the calculated opacity over these regions of multiple emission lines $\left(\lambda_{B} 404, \lambda_{B} 328\right)$ suggests a strong dominance of EUV photons in attenuating $\gamma$-rays even more than the $\mathrm{Ly}_{\alpha}$ line, as can be seen in Figure 2. The peak opacity value of the $\mathrm{Ly}_{\alpha}$ line was $\sim 14.0$ at $\sim 43.0 \mathrm{GeV}$, and the $\lambda_{B} 404$ and $\lambda_{B} 328$ were $\sim 70.9$ and $\sim 46.4$ at $\sim 13.5 \mathrm{GeV}$ and $\sim 10.8 \mathrm{GeV}$, respectively. Using the delta function approximation in equation (2.8), the opacity of $\mathrm{Ly}_{\alpha} \sim 16.5$, while it is $\sim 15.4$ when using equation (2) from the $[4,6]$ papers. Note that the absorption of the $\gamma$-ray photons depends on the location of the $\gamma$-ray emitting zone in the BLR. In case the $\gamma$-ray emitting zone is located outside or closer to the outer edge of the BLR, the opacity would be negligible or a fraction of the values we calculate here [11]. In addition to $e^{ \pm}$pair-production-related spectral breaks, the spectra of many blazars show dips in the $\gtrsim 10 \mathrm{GeV}$ range as can be seen in the literature $[4,6,13]$. It seems, therefore, reasonable that the lines that have significant absorption at $E \sim 50 \mathrm{GeV}$ would 
cause the dips observed beyond the spectral break of blazars. The calculated opacity of the BLR of blazars opens the possibility of constraining the emission zone of the $\gamma$-rays produced in the jets of AGN via the transmission function. We suggest that by modeling the BLR and including Galactic background radiation and their respective opacities it will be possible to locate the $\gamma$-ray emission zone.

\section{Acknolwdgements}

We extend our gratitude to R. C. Telfer for providing the ASCII data files of the composite spectra from [8]. This work was supported by grants from the National Research Foundation (South Africa).

\section{References}

[1] W. Zheng, G.A. Kriss, R.C. Telfer, J.P. Grimes and A.F. Davidsen, A composite HST spectrum of quasars, The Astrophysical Journal 475(2) (1997) p.469

[2] M. C. Urry and P. Padovani, Unified schemes for radio-loud active galactic nuclei, Publications of the Astronomical Society of the Pacific, 107(715) (1995) p.803.

[3] B. Czerny and K. Hryniewicz, The origin of the broad line region in active galactic nuclei, Astronomy \& Astrophysics 525 (2011) p.L8.

[4] J. Poutanen and B. Stern, GeV breaks in blazars as a result of gamma-ray absorption within the broad-line region, The Astrophysical Journal Letters 717(2) 2010 p.L118.

[5] H. Netzer and A. Laor, Dust in the narrow-line region of active galactic nuclei, The Astrophysical Journal 404 (1993) pp.L51-L54.

[6] B.E. Stern and J. Poutanen, The mystery of spectral breaks: Lyman continuum absorption by photon-photon pair production in the Fermi GeV spectra of bright blazars, The Astrophysical Journal 794(1) (2014) p.8.

[7] R.J. Gould and G.P. Schréder, Opacity of the universe to high-energy photons, Physical Review 155(5) (1967) p.1408.

[8] R.C. Telfer, W. Zheng, G.A. Kriss and A.F. Davidsen, The rest-frame extreme-ultraviolet spectral properties of quasi-stellar objects, The Astrophysical Journal 565(2) (2002) p.773.

[9] D.E. Vanden Berk, G.T. Richards, A. Bauer, M.A. Strauss, D.P. Schneider, T.M. Heckman, D.G. York, P.B. Hall, X. Fan, G.R. Knapp, and others, Composite quasar spectra from the sloan digital sky survey, The Astronomical Journal 122(2) (2001) p.549

[10] G. Ghisellini and F. Tavecchio, Canonical high-power blazars, Monthly Notices of the Royal Astronomical Society 397(2)(2009) pp. 985-1002

[11] R.J. Britto, S. Razzaque and B. Lott, Spectral Studies of Flaring FSRQs at GeV Energies Using Pass 8 Fermi-LAT Data, arXiv preprint arXiv:1502.07624 (2015)

[12] R.J. Gould and G.P. Schréder, Pair production in photon-photon collisions, Physical Review 155(5) (1967) p.1404.

[13] L. Costamante and S. Cutini and G. Tosti and E. Antolini and A. Tramacere, On the origin of gamma-rays in Fermi blazars: beyondthe broad-line region, Monthly Notices of the Royal Astronomical Society, 477(4) (2018) pp. 4749-4767. 